\title{
P I 4-03. HIV-specific T-lymphocyte proliferative responses induced by a multigene multiclade HIV-I DNA/MVA heterologous vaccine in Tanzanian volunteers
}

\author{
S Aboud*1, F Mhalu ${ }^{1}$, M Bakari ${ }^{1}$, J Lifson ${ }^{2}$, E Lyamuya ${ }^{1}, \mathrm{M}$ Marovich ${ }^{3}$, J Cox ${ }^{3}$, \\ E Sandstrom ${ }^{4}$, G Biberfeld ${ }^{5}$ and C Nilsson ${ }^{6}$
}

\begin{abstract}
Address: ${ }^{1}$ Department of Microbiology and Immunology, Muhimbili University of Health and Allied Sciences, Dar es Salaam, Tanzania, ${ }^{2}$ SAIC Frederick, Inc., National Cancer Institute at Frederick, Frederick, USA, ${ }^{3}$ US Military HIV Research Program, Rockville, MD, USA, ${ }^{4}$ Venhälsan, Karolinska University Hospital, Stockholm, Sweden, ${ }^{5}$ Swedish Institute for Infectious Disease Control and Karolinska Institute, Stockholm, Sweden and ${ }^{6}$ Swedish Institute for Infectious Disease Control (SMI), Stockholm, Sweden

* Corresponding author
\end{abstract}

from AIDS Vaccine 2009

Paris, France. 19-22 October 2009

Published: 22 October 2009

Retrovirology 2009, 6(Suppl 3):PI9| doi:I0.II86/I742-4690-6-S3-PI9I

This abstract is available from: http://www.retrovirology.com/content/6/S3/PI9I

(C) 2009 Aboud et al; licensee BioMed Central Ltd.

\section{Background}

A phase I/II HIV vaccine trial (HIVIS 03) employing a multiclade, multigene HIV-1 DNA prime/MVA boost vaccine among healthy volunteers is ongoing in Dar es Salaam, Tanzania.

\section{Methods}

Sixty healthy HIV negative volunteers including 15 females, were randomised to 3 groups and were injected at months 0,1 and 3 with plasmid DNA vaccine (produced by KI/SMI and Vecura, Sweden) containing gp160 of HIV-1 subtypes A, B, C; rev B; p17/p24 gag A, B and Rtmut $B$, at $1 \mathrm{mg}$ i.d. $(\mathrm{n}=20)$ or $3.8 \mathrm{mg}$ i.m. $(\mathrm{n}=20)$ or placebo $(n=20)$ using the Biojector. At month 9, a single i.m. injection with MVA $10^{8}$ pfu of MVA/CMDR expressing HIV-1 genes env, gag, pol of CRF01A_E (produced by NIAID and WRAIR, USA) or placebo was administered. Tlymphocyte proliferative (TLP) responses to AT-2 inactivated $H I V-1$ antigen from virus isolates/clade $M N / B$, KNH1144/A, TZA125/C and CM235/A_E were tested by a standard ${ }^{3} \mathrm{H}$-thymidine uptake assay. TLP was reported as stimulation index (SI) and SI above 6 was considered positive based on mean reactivity at baseline in 40 volunteers.

\section{Results}

Two weeks after the 3rd DNA/placebo injection, 24 of 52 (46\%) volunteers had positive TLP responses. Two weeks and two months after the HIV-MVA/placebo boost, 35 of $48(73 \%)$ and 32 of 44 (73\%) volunteers had positive TLP responses, respectively. Thus far, 33 volunteers have been tested six months after the HIV-MVA/placebo boost and $20(61 \%)$ have positive TLP responses. There was a high degree of cross reactivity as shown by strong proliferative responses to all four isolates tested. The TLP responses were sustained and 6 months after the HIV-MVA boost the greatest reactivity was seen against CM235/A_E. The study remains blinded.

\section{Conclusion}

HIVIS03 DNA-MVA vaccine has so far demonstrated strong HIV-specific T-lymphocyte proliferative responses with a high degree of cross clade reactivity. 